\title{
Towards In-flight Measurements of Helicopter Blade Tip Vortices
}

\author{
Kolja Kindler ${ }^{1,2}$, Karen Mulleners ${ }^{1}$, and Markus Raffel ${ }^{1}$ \\ 1 German Aerospace Centre (DLR), Bunsenstrae 10, 37073 Göttingen, Germany \\ 2 Present address: Max Planck Institute for Marine Microbiology, Celiusstr. 1, \\ 28359 Bremen, kkindler@mpi-bremen.de
}

\begin{abstract}
In the framework of the AIM project the near field of the blade tip vortex of a full-scale helicopter in simulated hover flight was investigated by combining three-component Particle Image Velocimetry and Background Oriented Schlieren measurements. The velocity field measurements in the range of wake ages of $\psi_{v}=1^{\circ}$ to $30^{\circ}$ in azimuth provided a reference for a quantitative analysis of the Schlieren results yielding vortex core density estimates. Ongoing vortex roll-up was observed at $\psi_{v}=1^{\circ}$ while considerable aperiodicity was persistent thereafter. The vortex parameters for $\psi_{v}>1^{\circ}$ were consistent with the Scully vortex model. However, the particular challenges of full-scale, outdoor testing, especially the limited spatial resolution and aperiodicity effects, yielded a higher level of measurement uncertainty as compared to sub-scale experiments.
\end{abstract}

\subsection{Introduction}

The blade tip vortices trailing from the main rotor of a helicopter are considered the dominant coherent structures of the rotor wake, especially, with respect to fluid-structure interaction, aeroacoustics, etc.. As such, the blade tip vortex has drawn considerable attention in terms of analytical, experimental, and increasingly numerical investigations during the past decades [3].

Due to the inherent complexity of the helicopter flow field, wind tunnel experiments as well as numerical investigations by means of computational fluid dynamics (CFD) are rather intricate, time-consuming, and cost-intensive [13]. Furthermore, the relevant dimensionless parameters, such as e.g. the reduced vortex Reynolds numbers $R e=\Gamma_{v} / \nu$ (with the circulation $\Gamma_{v}$ and the kinematic viscosity $\nu$ ), obtained in sub-scale experiments are often not in accordance with the full-scale quantities. Hence, characteristic aerodynamic scaling is exacerbated. Therefore, it is appealing to address the total system and to adapt and implement available experimental methods to probe the blade tip vortex characteristics in situ, i.e. in flight. Full-scale, in-flight experiments provide access to a much larger range of flight parameters than typically attainable in wind tunnel testing. Thereby, complex aerodynamic 


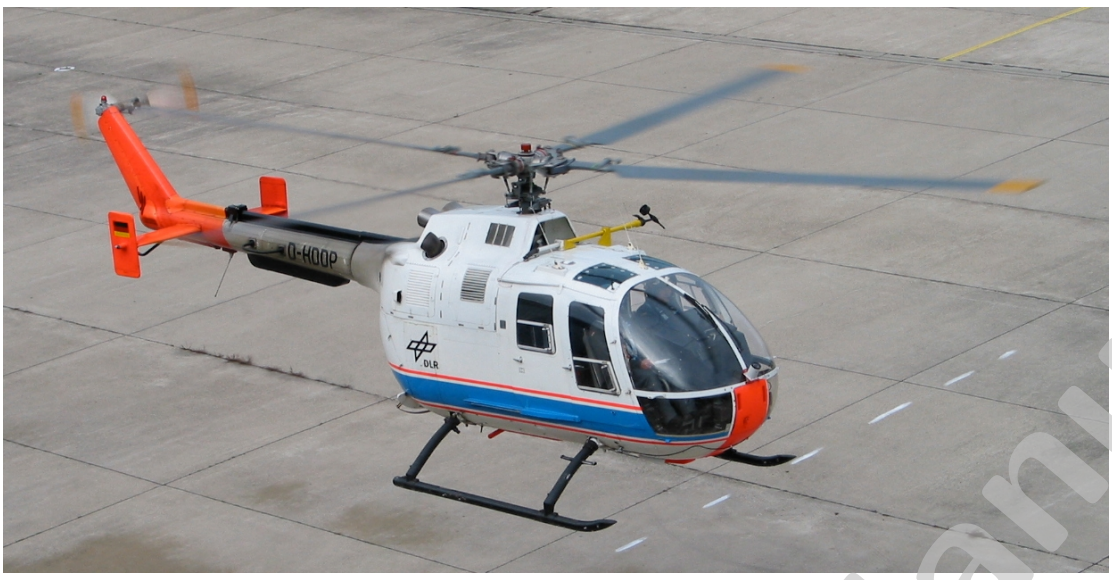

Fig. 1.1. DLR's MBB Bo105 test helicopter.

problems such as blade-vortex interaction and tip vortex control can be directly addressed. To this context, the near field of the tip vortex ${ }^{3}$ is of particular importance since measures of active and passive vortex control target this crucial stage of development. Despite its significance, only few experimental reports addressing full-scale blade tip vortices $[2,4,21]$ consider the near field.

Hitherto, most of the sub-scale experiments have utilised Laser Doppler Velocimetry (LDV) (e.g. [11,12]). Although providing a high measurement accuracy, LDV requires an independent determination of the vortex centre position in relation to the measurement volume. Therefore, it is inadequate for in-flight testing. Particle Image Velocimetry (PIV) on the other hand, captures an entire region around the vortex which allows for a precise relation of swirl velocities to the vortex centre (see e.g. [22] and references therein). However, the application of PIV, as well as LDV, for ground independent testing would require cumbersome instrumentation to be integrated into the helicopter.

A promising technique for in-flight velocity field investigations is Light Detection and Ranging (LIDAR) which is reported elsewhere in this volume. While the technical implementation of a LIDAR system in a helicopter is reasonably practicable, the supply of tracer particles in the measurement area is rather challenging.

Aside LIDAR, Background-oriented Schlieren (BOS) appears to be the most practical method for efficient full-scale in-flight helicopter investigations. The BOS technique is based on visualising light deflections caused by density gradients within the flow field. In order to quantify these deflections artificial speckle pattern are imaged with and without an aerodynamic structure in-

${ }^{3}$ The near field denotes the region directly behind the blade tip where the initial development of tip vortex takes place. 
fluencing the light path. The vortex structure is subsequently reconstructed by cross-correlating the image-reference pairs $[15,20]$. BOS is particularly suitable for ground independent testing because natural formations such as grass, cornfields, and skirts of forests provide adequate backgrounds $[8,9,17]$. In the framework of the AIM project a BOS sensor unit was developed and certified which integrates a digital high-resolution camera capable of rotor synchronised image acquisition into the helicopter.

The present investigation includes BOS measurements of the near field of the blade tip vortices. Beyond qualitative vortex visualisation, quantitative density field information can be derived from the monoscopic BOS data if rotational symmetry of the vortical structures can be presumed $[6,9,25]$. However, rotational symmetry of the blade tip vortex within the near field cannot be expected a priori. Therefore, a reference measurement of the vortex velocity field in simulated hover flight, i.e. with the helicopter fixed on the ground, was carried out by means of PIV. Based on three component PIV data at vortex ages of $\psi_{v}=1^{\circ}$ to $30^{\circ}$ deviations from rotational symmetry within the vortex formation stage were evaluated and constraints for the BOS results were defined. This investigation was intended to lay the foundations for future fully ground-independent, in-flight measurements including tip vortex visualisation and, to a certain extend, to provide quantitative core density estimations.

\subsection{Experimental Methods}

\subsubsection{Test helicopter}

For this study DLR's MBB Bo105 experimental helicopter was fixed on the ground and operated in simulated hover flight in ground effect. The fixation of the helicopter is experimentally most favourable and the development of tip vortices within the range of wake ages relevant to this study can be anticipated to be well defined. The main rotor of the test helicopter had $N_{b}=4$ hingeless blades of rectangular planform with a radius of $R=4.91 \mathrm{~m}$, a chord length of $c=0.27 \mathrm{~m}$, a solidity of $\sigma=N_{b} c /(\pi R)=0.07$, and $-8^{\circ}$ linear blade twist. The angular velocity of the main rotor was $\Omega=44 \mathrm{rad} / \mathrm{s}$ yielding a blade tip Mach number of $M a=0.64$. During simulated hover flight, the helicopter generated approximately $T=20000 \mathrm{~N}$ thrust corresponding to a thrust coefficient of $C_{T}=T /\left(\rho \pi \Omega^{2} R^{4}\right)=0.0046$ resulting in a blade loading of $C_{T} / \sigma \simeq 0.066$.

\subsubsection{Particle Image Velocimetry}

Measurement configuration. The measurement plane was located on the port-side, parallel to the trailing edge of the blade (cf. figure 1.2). The PIV

data acquisition system consisted of two $10.7 \mathrm{Mpx}$ CCD cameras equipped with $300 \mathrm{~mm}$ objectives in a stereoscopic configuration. The cameras were 


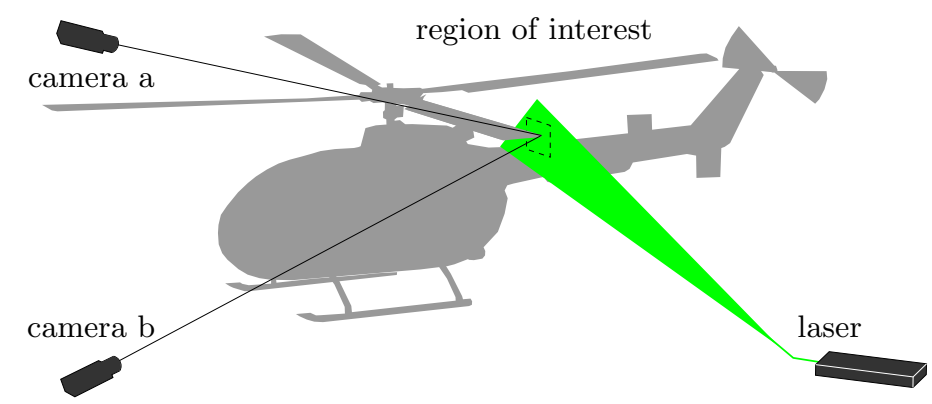

Fig. 1.2. Experimental set-up for stereoscopic PIV measurements of the blade tip vortex.

positioned on a vertical support ca. $1.5 \mathrm{~m}$ above and $2.1 \mathrm{~m}$ below the rotor plane in idle condition at a distance of $10 \mathrm{~m}$ away from the observation area. Illumination of the flow field was provided by means of a laser light sheet fed by a double cavity Nd:Yag pulse laser with a wave length of $532 \mathrm{~nm}$ and an energy of $280 \mathrm{~mJ}$ per pulse. The laser sheet was oriented parallel to the trailing edge of the blade such that the tip vortices are likely to be measured in a plane normal to their axis. The spatial resolution of the image acquisition system was $4 \mathrm{px} / \mathrm{mm}$ (corresponding to $1080 \mathrm{px} / \mathrm{c}$ ) for a field of view (FOV) of approximately $0.9 \mathrm{~m} \times 0.6 \mathrm{~m}$. The PIV system was synchronised with the main rotor taking advantage of an inductive rotor position indicator permanently installed for rotor balancing purposes.

When it comes to on-board, in-flight PIV applications, the provision of a homogeneous and sufficiently dense tracer particle distribution is an extremely demanding task. The atmospheric background conditions, i.e. even moderate cross-winds, might strongly alter the optimal injection point and, therefore, the tracer density within the field of view. Hence, the tracer generation and supply including an injection nozzle reaching approximately $3 \mathrm{~m}$ above the rotor disk, were realised on a mobile platform which could be relocated in response to the outer conditions at a distance of one rotor radius apart from the rotor disk. During the tests, the ambient conditions were close to the international standard atmosphere at an average temperature and atmospheric pressure of $10^{\circ} \mathrm{C}$ and $1015 \mathrm{hPa}$, respectively, with calm winds below $1.5 \mathrm{~m} / \mathrm{s}$ and intermittent gusts of less than $4 \mathrm{~m} / \mathrm{s}$.

Data evaluation methodology. The stereoscopic PIV data were evaluated according to standard procedures [18]. The intensity images acquired were high-pass filtered and normalised using the series minimum image prior to further processing. A camera view misalignment correction was computed for each time series as part of the image de-warping to compensate for small 
image offsets which would be greatly amplified due to the large scale geometry of the set-up [16].

To optimise the interrogation window size and overlap, the scheme of Richard $\&$ van der Wall [19] was followed. The interrogation window size was minimised with respect to an acceptable signal-to-noise ratio while the window overlap was maximised to avoid artificial smoothing of velocity gradients. The correlation analysis of the interrogation windows represents a low-pass filter limiting the peak velocities accessible [19]. By increasing the sampling window overlap, the probability of an interrogation window being perfectly centred at the maximum velocity increases, minimising spatial averaging effects by the interrogation window itself. In this case, rectangular cross-correlation windows of width $32 \mathrm{px}$ with an overlap of approximately $94 \%$ were used. The corresponding physical resolution, the ratio of the PIV measurement volume to chord length, is $L_{m} / c=0.0296$ which translates into $L_{m} / r_{c}=0.593$ using $r_{c}=0.05 c$ as an estimate of the vortex core radius. This value is at the lower range of numerous recent investigations ranging from $L_{m} / r_{c}=0.19$ to 2.6 (cf. [23] and references therein) but is at least a factor of 3 coarser than the commonly accepted resolution requirement of $L_{m} / r_{c}<0.2$ [14]. However, the true resolution is determined by the resulting vortex radius depending on vortex age. Additionally, a multi-grid evaluation scheme is used within the correlation analysis, in order to increase the resolution of regions of strong shear.

Prior to further analysis, the averaged background velocities within the observation area were subtracted from the instantaneous velocity fields, which mainly affected the out-of-plane component and does not interfere with the vortex centre identification. Subsequently, the vortex centres were identified using the scalar function introduced by Graftieaux et al. [7] in its discrete form

$$
\Gamma_{1}(P)=\frac{1}{N} \sum_{S} \frac{\left(P M \times U_{M}\right) \hat{e}_{x}}{\|P M\| \cdot\left\|U_{M}\right\|}
$$

where $N$ is the number of points in the two-dimensional neighbourhood $S$ of any given point $P$ in the $y, z$ plane, $M$ lies in $S, \hat{e}_{x}$ is the unit vector in $x$ direction, and $U_{M}$ is the in-plane velocity at $M$. The extremum of $\Gamma_{1}$ is identified with the vortex centre ${ }^{4}$. Unlike alternative criteria for vortex centre identification such as vorticity peak detection, $\lambda_{2}$, etc., the $\Gamma_{1}$ function does not require velocity field derivatives or the out-of-plain component reconstructed during evaluation and, therefore, is less susceptible to experimental noise (for a comprehensive review of the robustness of a variety of gradient-based operators and velocity field as well as convolution based methods it should be referred to $[5,23])$. Especially, large-scale PIV measurements of unsteady flow fields tend to yield spiky velocity derivatives leading to less reliable vortex centre identification than the scheme based on equation 1.1.

\footnotetext{
${ }^{4}$ Note that $\Gamma_{1}$ is not to be confused with $\Gamma_{v}$ the circulation of a vortex.
} 

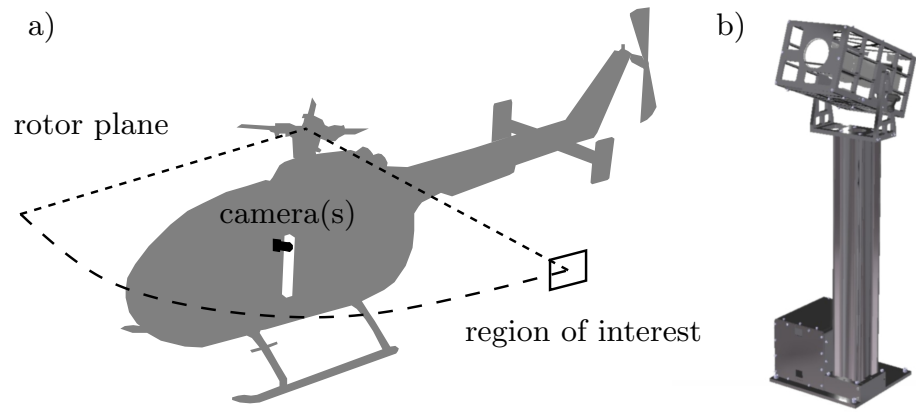

Fig. 1.3. Sketch of the airborne stereoscopic BOS imaging system as used for preliminary investigations (a); the sensor unit designed and certified for rotor-synchronised in-flight measurements (b).

Finally, the inclination of the vortex axis with respect to $y$ and $z$ spanning the measurement plane were estimated based on the out-of-plane plane velocity gradients $(\partial u / \partial y, \partial u / \partial z)$ outside two times the core radius and the ratio of maximum swirl and core radius $V_{s, \max } / r_{c}$ using an iterative procedure given by [23]. The inclination angles were found to be smaller than $3.5^{\circ}$ for the data relevant to this study, which is considered negligible, rendering a transformation into the vortex system unnecessary.

Measurement accuracy assessment. The data quality of the full-scale PIV measurements is found to vary strongly for subsequent time steps. The measurement noise level is considerably higher than typically achieved in laboratory experiments. Obviously, these variations are primarily due to the varying or intermittently inhomogeneous tracer concentration including tracer density gradients within the region of interest. Due to the unsteady nature of the flow field dissociated tracer patches of variable size are commonly found. Because the tracers are injected from above the rotor plane, the downwash tends to induce higher tracer concentrations inboard of the blade tip, while the fluid from the recirculation region outboards features stronger patchiness and, on average, lower tracer concentration.

The image pairs where the lack of tracers resulted in non-physically dysmorphic or undetectable vortex structures in the velocity fields were excluded from the analysis. As a result, approximately $10 \%$ of the series of 120 velocity fields at each wake age were discarded. The local tracer density represented the intrinsic limit of measurement resolution and accuracy as it defines the minimal interrogation window size and the validity of the results. 


\subsubsection{Background-oriented Schlieren}

The BOS sensor unit. The sensor unit for the initial measurements consisted of two high-resolution Canon OES 1Ds Mark II cameras with a focal length of $f=70$ to $200 \mathrm{~mm}$ mounted on an optical rail placed inside the helicopter (figure 1.3a). Images were acquired at $f=200 \mathrm{~mm}$, corresponding to an f-number of $f_{\#}=f / 22$ at a resolution of $0.169 \mathrm{~mm} / \mathrm{px}$.

Based on the lessons learned from the initial measurements, an advanced in-flight BOS sensor unit was developed, manufactured and certified (figure 1.3b). The sensor consisted of the same camera and lens as above cased in an aluminium box which is pivotable in the vertical. The box was supported by a standard optical rail mounted on an available base plate behind the copilot's seat of the helicopter. Additionally, the construction featured a second casing for trigger electronics and power supply. The acquisition of image series at constant frame rate was initiated by a trigger signal synchronised with the main rotor. The camera was remotely controlled by a portable computer which did not have to included in the certification process.

Measurement configuration. The background was provided by an artificial varicoloured speckle pattern of $4 \times 4 \mathrm{~m}$ in size attached to the front of a hangar approximately $5 \mathrm{~m}$ above ground. The distance between the camera and the expected tip vortex position was approximately $R$, while the distance to the background was $5 R$. The sampling frequency was $4 \mathrm{~Hz}$ yielding a sufficient inter-framing time for the vortex to be shifted to an initially undisturbed area of the image. The image displacement, $\delta z$, measured using an artificial background at a distance of $1.5 R$ from the vortex showed a symmetrical structure (figure 1.4). The interrogation window size was $36 \mathrm{px} \times 36 \mathrm{px}$ with a $50 \%$ window overlap and the global uncertainty, in this case, was less than $10 \%$ of the deflections. Atmospheric conditions close to the standard atmosphere at $24^{\circ} \mathrm{C}$ were considered.

Schlieren measurement accuracy. Since the deviations of the apparent pixel shift, $\delta z$, are mainly of random nature uncertainties can be expected to be averaged out during subsequent integration. The systematic error of the deviation angle, $\Delta \gamma$, was due to uncertainties of the magnification, $m$, and the distance between the vortex core and background, $a$, and is given by

$$
\Delta \gamma=\left|-\frac{\delta z}{m^{2} a} \Delta m\right|+\left|-\frac{\delta z}{m a^{2}} \Delta a\right| .
$$

These errors caused a constant deviation of $\gamma$ which translates into a constant deviation of the tomographic reconstruction. The density uncertainties specified below were estimated based on the relative errors of $\gamma$. 

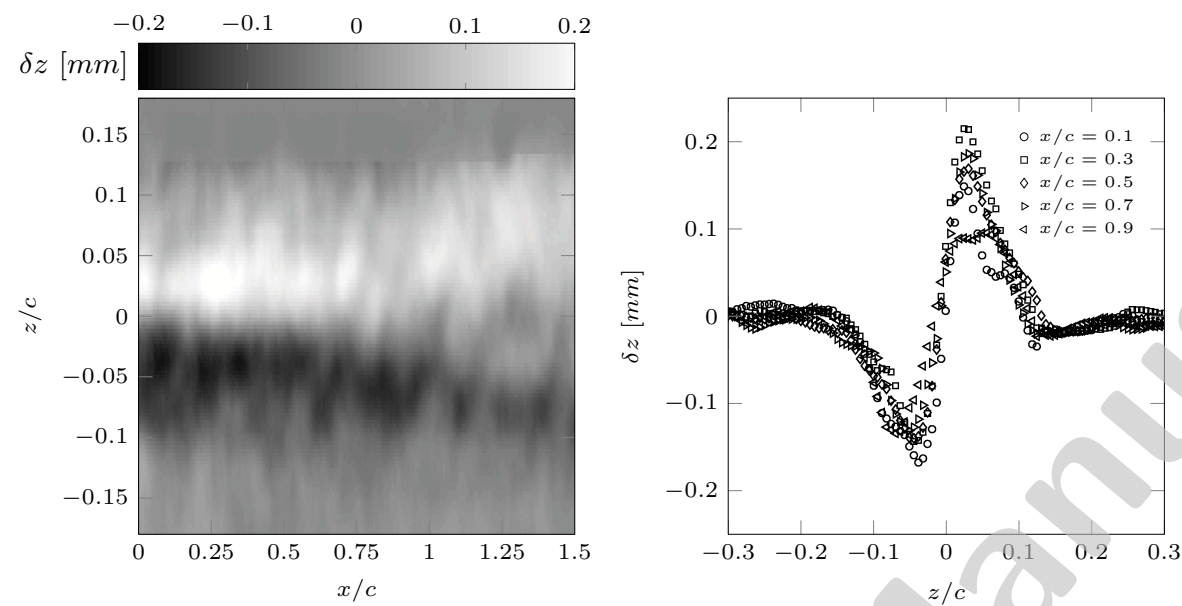

Fig. 1.4. Image displacement $\delta z$ and displacement profiles extracted at $x / c=0.1$, $0.2,0.50 .7$, and $0.9\left(\psi_{v}=0.3^{\circ}, 0.6^{\circ}, 1.6^{\circ}, 2.2^{\circ}\right.$, and $\left.2.8^{\circ}\right)$ downstream from the trailing edge of the blade.

\subsection{Results and Discussion}

\subsubsection{Average vortex velocity fields}

With the exception of vortex ages of $\psi_{v}<5^{\circ}$, the conditionally averaged velocity and the corresponding vorticity fields of the blade tip vortex indicated a spherical cross-section while the $z$-component, i.e. the cross-flow component, exhibits noticeable stretching in $y$ (figure 1.5). This departure from circularity is associated with the minor inclination of the vortex axes with respect to the measurement plane. The extension of the cross-flow field to the lower left represents the drag bucket behind the rotor blade. Due to the blade circulation exhibiting a strong gradient towards the tip, a sheet of trailed vorticity is shed into the wake, which is recognisable in the vorticity distribution from the vortex centre down to the left of the image.

\subsubsection{Vortex formation stage}

In order to trace effects of vortex formation, i.e. shear layer roll-up, the rotational symmetry of the vortex velocity fields was considered. Approximating $\Gamma_{1}$ by a bivariate Chauchy distribution of the form

$$
L=\frac{a}{1+\frac{x^{2}}{b}+\frac{y^{2}}{c}},
$$

where $a, b$, and $c$ are fit parameters, the principle axes were derived and the vortex circularity was expressed as the principal axes ratio 


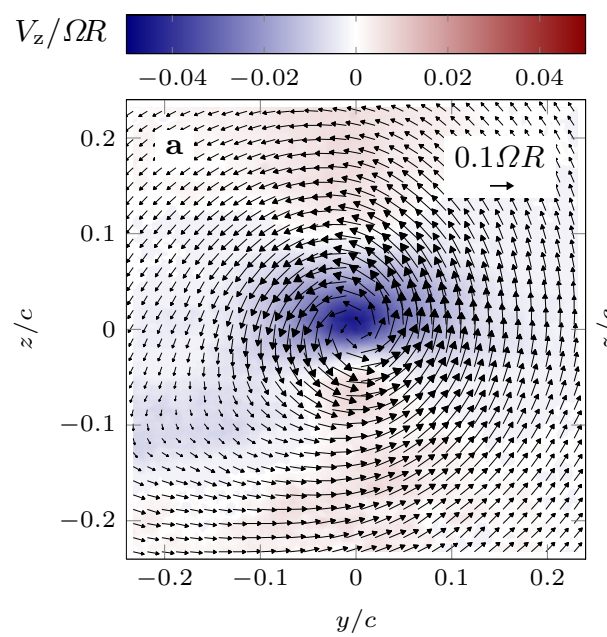

$\omega_{\mathrm{z}} / \Omega$
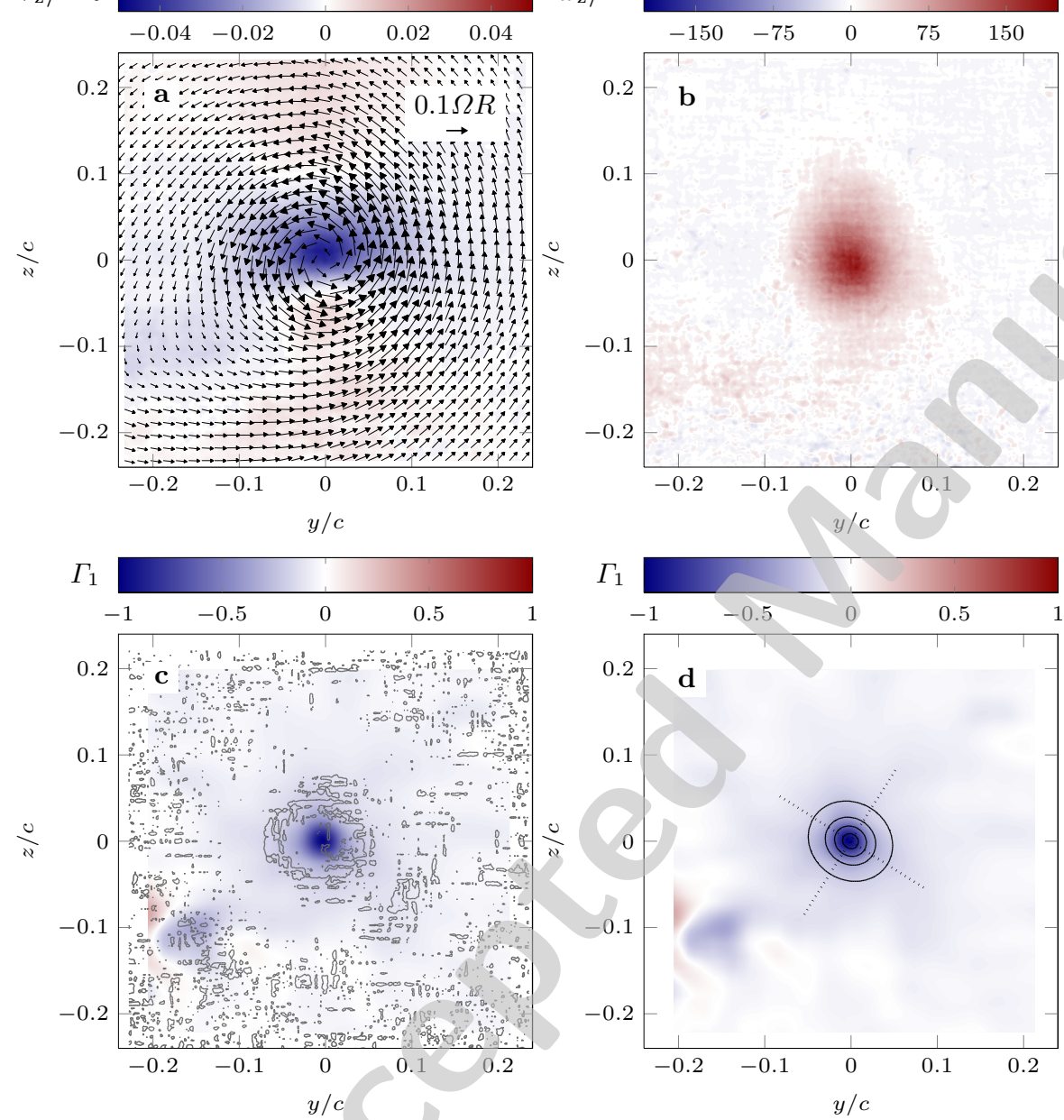

Fig. 1.5. The conditionally averaged velocity field $\psi_{v}=5^{\circ}$ (a) and the corresponding in-plane vorticity component $\omega_{x}$ (b). A comparison of $\Gamma_{1}$ with the zero-crossing of $\lambda_{2}$ (c) and the circularity of the vortex cross-section derived from $\Gamma_{1}$ for a representative instantaneous field at $\psi_{v}=5^{\circ}(\mathrm{d})$. Velocities are scaled by the blade tip speed $\Omega R$, the in-plane component of vorticity scaled by the angular velocity of the main rotor, $\Omega$.

$$
\epsilon=\sqrt{1-\frac{b}{c}}
$$

Additionally, the inclination angle of the major axis with respect to the horizontal was extracted.

The distribution of the axis ratio indicated noticeable asymmetry of the vortices which decreased with increasing wake age (figure 1.6a). At the 

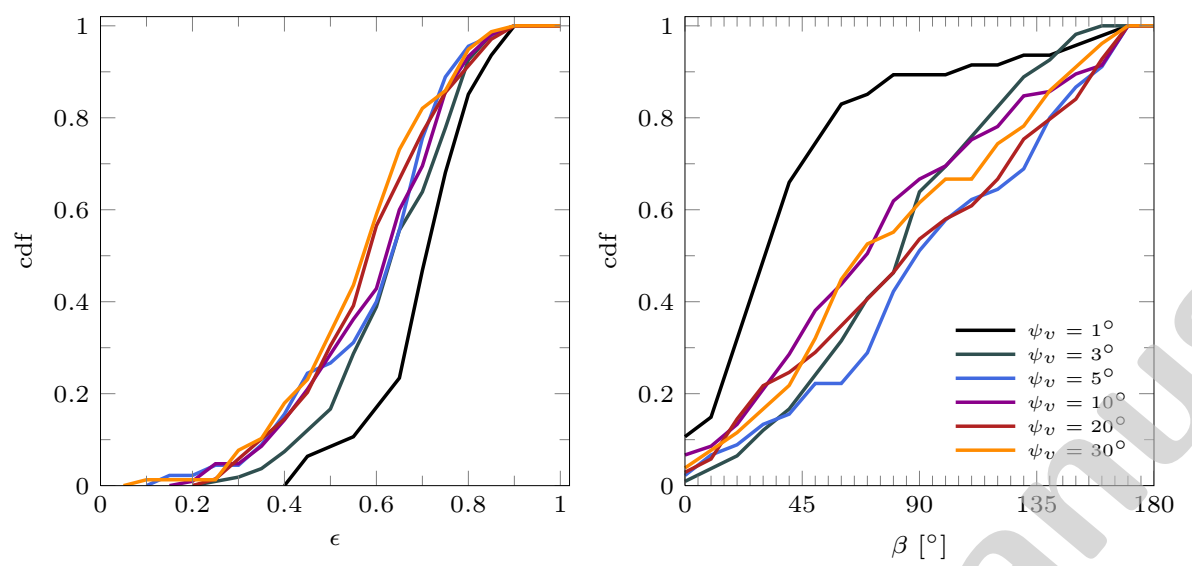

Fig. 1.6. Cumulative propability density of the principal axis ratio $\epsilon$ and the inclination angle of the major axis $\beta$.

same time, the inclination angles $\beta$ were uniformly distributed irrespective of $\psi_{v}=1^{\circ}$ (figure $1.6 \mathrm{~b}$ ). For the youngest wake age considered the major axis had a preferential orientation with respect to the horizontal which was associated with the predominantly spiral structure in this range of development. Downstream from the initial vortex formation stage, noticeable aperidicity of the vortex velocity fields indicated incomplete relaxation of the cores. In the range of wake ages considered, aperiodicty effects imposed the major constrain to axial symmetry which contributed to the elevated level of uncertainty of the velocity vortex profiles [10].

\subsubsection{Low-order vortex approximation}

In order to facilitate a mutual comparison of the BOS and PIV results, the velocity fields were approximated by a low-order vortex velocity model [24], which is outlined below for convenience. The non-dimensional tangential, radial, and axial velocity take the form

$$
\begin{gathered}
\bar{V}_{\Theta}=\frac{\bar{r}}{\left(1+\bar{r}^{2 n}\right)^{1 / n}}, \\
\bar{V}_{r}=-\frac{2(1+n) \bar{r}^{2 n-1}}{1+\bar{r}^{2 n}}, \\
\bar{V}_{x}=\frac{4 n(1+n) \bar{r}^{2(n-1)}}{\left(1+\bar{r}^{2 n}\right)^{2}} \bar{x},
\end{gathered}
$$

where $\bar{V}_{\Theta}=V_{\Theta} / V_{\Theta, 0}$ with $V_{\Theta, 0}=\Gamma_{v} / 2 \pi r_{c}, \bar{r}=r / r_{c}, \bar{V}_{r}=V_{r} r_{c} / \nu, \bar{V}_{x}=$ $V_{x} r_{c} / \nu$, and $\bar{x}=x / r_{c} . \Gamma_{v}$ is the circulation of the vortex, $\nu$ is the dynamic 

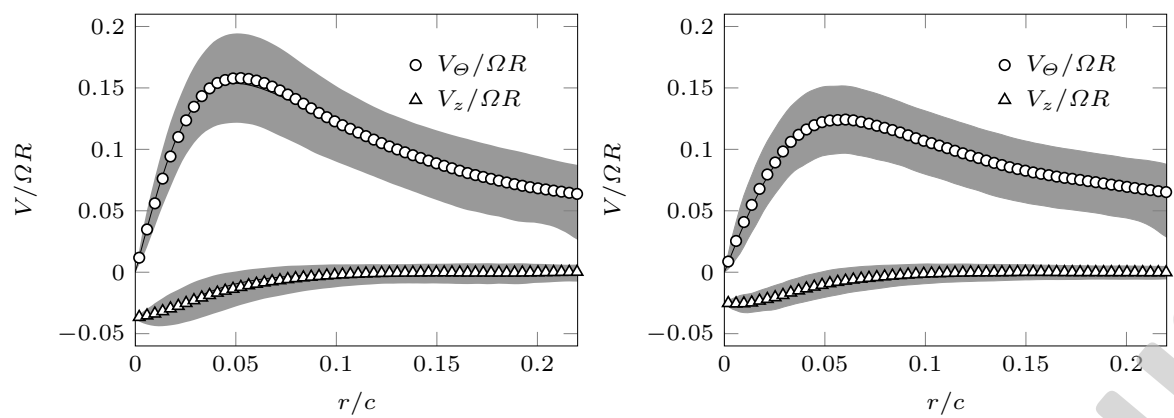

Fig. 1.7. Velocity profiles of blade tip vortex at $\psi=3^{\circ}$ (left) and $\psi=30^{\circ}$ (right); the shading denotes the standard deviation of the measured data, lines represent the best fit to equation 1.5 and 1.7 using $n=1$ (Scully vortex).

viscosity of air. The core radius $r_{c}$ and $V_{\Theta, 0}$ were determined by least-square fitting. Treating $n$ initially as free parameter to be identified, yielded values very close to $n=1$ corresponding to the Scully vortex model. Hence, this model was applied in the remainder of the evaluation, reducing the degrees of freedom of the parameter identification to $\Gamma_{v}, r_{c}$, and $\bar{x}$.

The tangential, radial, and axial velocity profiles were azimuthally averaged with respect to the vortex axis prior to conditionally averaging the time series which were then approximated by the model (figure 1.7). Both the tangential and axial velocity, $V_{\Theta}$ and $V_{x}$, are consistent with the model of equation 1.5, and 1.7, where the standard deviation is below $0.03 \Omega R$. The weak radial velocity component $V_{r}$ (not shown) is found to vanish to within the measurement accuracy. The radial component is particularly sensitive to effects of lateral vortex convection and background effects. Additionally, the downwash contributes to depressing the radial velocities inboard of the tip affecting the axial average of the velocity profiles. This is especially the case for a hovering rotor, where at the inboard side of the vortex a strong mean downwash is superimposed on the vortex velocity field while at the outboard side no such downwash is present. In forward flight, the mean downwash is significantly smaller and this effect is largely alleviated.

The average vortex core radii for $\psi \geq 3^{\circ}$ were in the range $\bar{r}=0.04$ to 0.06 with peak tangential velocities of $\bar{V}_{\Theta}=0.15$ to 0.12 . In absence of any reference near field data these results cannot be validated. However, earlier full-scale measurements resulted in peak swirl velocities of $\bar{V}_{\Theta}=0.2$ to 0.5 and initial core radii of $\bar{r}=0.02$ to 0.03 in the far field. This is in agreement with recent sub-scale measurements [19] although $R e_{v}$ is only half as large. The reasons for this difference are manifold. First, the persistent residual asymmetry indicates that the vortex roll-up is incomplete within the wake age interval investigated. In this area the initial core size is related to the boundary layer thickness close to blade tip, which in turn depends on the tip 
Mach number, angle of attack, etc.. The deviations from rotational symmetry were translated into reduced tangential velocities and increased core radii by the averaging procedure applied. Furthermore, straining effects which promote concentration of the core are known to take effect at larger wake ages. The overall increased measurement noise level caused additional smoothing of the steep velocity gradients.

\subsubsection{Vortex core densities}

The vortex core density fields were reconstructed from the Schlieren results using a method based on an inverse Radon transform according to [9] (figure 1.8). Subsequently, the azimuthally averaged density profiles, were approximated by the Scully vortex model (equation 1.5 to 1.7). Additionally, the reduced density reads [1]:

$$
\frac{\rho}{\rho_{\infty}}=\left(1-\frac{\kappa-1}{1+\left(r / r_{c}\right)^{2}} \zeta\right)^{\frac{1}{1-\kappa}},
$$

assuming inviscid and isentropic flow. Here, $\kappa$ is the specific heat ratio and $\zeta$ is a fit-parameter together with $r_{c}$. The considerations were restricted to an area between $x / c=0$ to 1 , i.e. $\psi=0$ to $3^{\circ}$ where effects of vortex motion towards the fuselage were expected to be negligible.

The total density loss was approximately $25 \%$ at $x / c=0.1$ and decreased towards $10 \%$ at $x / c=0.9$. The best fit of equation 1.8 yielded $\bar{r}=0.043$, $0.049,0.055,0.054$, and 0.060 at $x / c=0.1,0.3,0.5,0.7$, and 0.9 . Due to the helicopter motion during the measurement, the relative uncertainty of the density data did not fall below $20 \%$ which translated into an estimated core radius deviations of $\Delta r_{c}=0.005$ without taking into account resolution effects. The reduced density exceeded $\rho / \rho_{\infty}=1$ at the core boundaries and relaxed radially. Although this behaviour is not included in the Scully model (equation 1.8) the fits were in satisfactory agreement with the measurements.

The averaged vortex radii determined from the three-component PIV measurements were found to be in agreement with the corresponding values derived from BOS measurements of the same experimental configuration as reported in [9]. At a wake age of $\psi_{v}=3^{\circ} \mathrm{PIV}$ and BOS yield a core radius of $\bar{r}=0.055$ and 0.060 , respectively, at a core density drop of $10 \%$ with respect to the ambient and a peak tangential velocity of $\bar{V}_{\Theta} / \Omega R=0.149$. It should be noted that at this particular vortex age the roll-up process was still incomplete. The conformity of the full-scale PIV and BOS results can partly be ascribed to the fact that both correlation based optical methods share similar limitations in terms of spatial resolution. However, the two independent experiments provide sufficient cross-validation to within the measurement accuracy. 


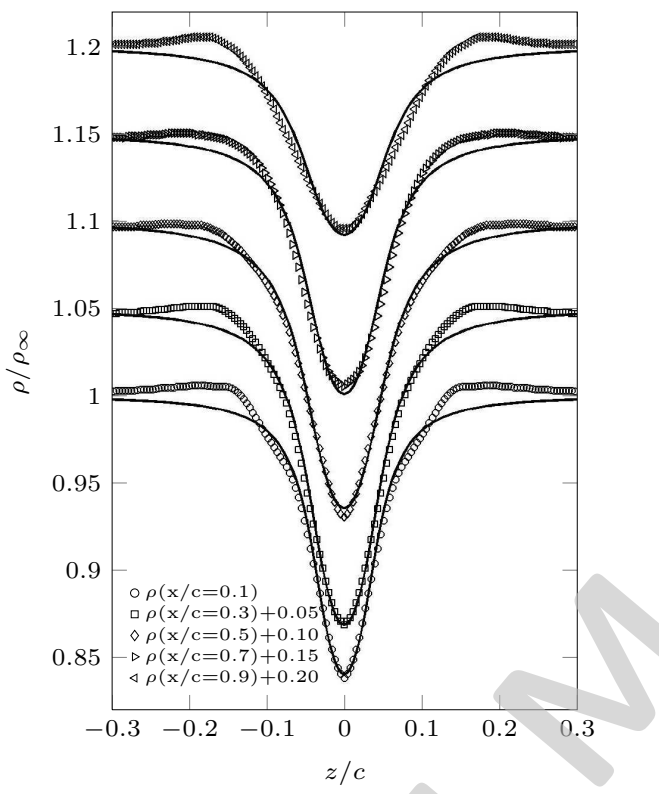

Fig. 1.8. Tomographic reconstruction of the density profiles at $x / c=0.1,0.3,0.5$, 0.7 , and $0.9\left(\psi_{v}=0.3^{\circ}, 0.6^{\circ}, 1.6^{\circ}, 2.2^{\circ}\right.$, and $\left.2.8^{\circ}\right)$. The solid lines represent the best fit of equation 1.8 .

\subsection{Conclusions}

An airborne rotor synchronised BOS image acquisition system was developed and certified to study the tip vortex characteristics in situ under various flight conditions. First BOS results in the near field yielded quantitative core density estimations, where rotational symmetry was assumed. To quantify effects of vortex formation in the near-field, three-component velocity measurements on a full-scale Bo105 helicopter in ground effect were performed as a reference measurement. The analysis of the range of of wake ages of $\psi_{v}=1^{\circ}$ to $30^{\circ}$ indicated sufficient rotational symmetry to be attained closely downstream ofrom the blade tip. Within the near-field, i.e. the vortex formation region, the measurement uncertainty was elevated. Due to persisten aperidocity of the vortex flow field, the peak swirl velocities were underestimated while core radii were overestimated. Thereafter, the average tangential and axial velocities were well described by the Scully vortex model. The vortex core radii derived from velocity and Schlieren measurements coincide to within measurement uncertainty.

Based on the results presented here a straightforward integration of an available measurement techniques such as BOS can be efficiently utilised for in-flight testing. Thereby, the range of accessible flight parameters can be expanded when compared to wind tunnel testing. Future efforts will include 
experiments probing the tip vortex trajectories in the far field as well as investigations of different measures of vortex control and their impact on the near and far field. Finally, blade-vortex interaction will be addressed within the full-scale system.

\section{Acknowledgements}

This work has been part of the European Commission funded project "Advanced In-Flight Measurement Techniques" (AIM) and the analysis has partly been performed in the framework of the US/German Memorandum of Understanding on Helicopter Aerodynamics, Task VIII "Rotor Wake Measurement Techniques". The authors are greatly indebted to F. Leopold from the FrenchGerman Research Institute of Saint-Louis and E. Goldhahn from the Institute for Turbo Machinery, University of Hannover for their valuable contributions to the realisation and analysis the Schlieren measurements. Furthermore, the dedicated support by the helicopter crew R. Gebhard and U. Göhmann as well as by our colleagues B. G. van der Wall, H. Richard, M. Jönsson ,and M. Kühn is gratefully acknowledged.

\section{References}

1. A. Bagai and J. G. Leishman. Flow visualization of compressible vortex structures using density gradient techniques. Exp. Fluids, 15:431, 1993.

2. D. W. Boatwright. Measurements of velocity components in the wake of a full-scale helicopter rotor in hover. Technical Report USAAMRDL Technical Report 72-33, U.S. Army Air Mobility Research and Development Laboratory Fort Eustis, Virginia, 1972.

3. A. T. Conlisk. Modern helicopter rotor aerodynamics. Progr. in Aerospace Sci., $37: 419,2001$.

4. C. V. Cook. The structure of the rotor blade tip vortex. In AGARD CP-111, 13-15 September, 1972.

5. R. Cucitore, M. Quadrio, and A. Baron. On the effectivenss and limitations of local criteria for the identification of a vortex. Eur. J. Mech. B. Fluids, $18(2): 261,1999$.

6. E. Goldhahn and J. Seume. Background oriented Schlieren technique - sensitivity, accuracy, resolution and application to three-dimensional density fields. Exp. Fluids, 43:241, 2007.

7. L. Graftieaux, M. Michard, and N. Grosjean. Combining PIV, POD and vortex identification algorithms for the study of unsteady turbulent swirling flows. Meas. Sci. Technol., 12:1422, 2001.

8. M. J. Hargather and G. S. Settles. Natural-background-oriented Schlieren imaging. Exp. Fluids, 48(1):59, 2009.

9. K. Kindler, E. Goldhahn, F. Leopold, and M. Raffel. Recent developments in background oriented Schlieren methods for rotor blade tip vortex measurements. Exp. Fluids, 43:233, 2007. 
10. K. Kindler, K. Mulleners, H. Richard, B.C. van der Wall, and M. Raffel. Aperiodicity in the near-field of full-scale rotor blade tip vortices. Exp. Fluids, 2010. in press.

11. J. G. Leishman. Principles of helicopter aerodynamics. Cambridge University Press, Cambridge, UK, 2001.

12. J. G. Leishman, A. M. Baker, and A. J. Coyne. Measurement of rotor tip vortices using three-component LDV. J. Am. Heli. Soc., 41(4):342, 1995.

13. J. W. Lim, T. A. Nygaard, R. Strawn, and M. Potsdam. BVI airloads prediction using CFD/CSD loose coupling. In 4th AHS Vertical Lift Aircraft Design Conference, San Francisco, CA, USA, January 16-20, 2006.

14. P. B. Martin, J. G. Pugliese, J. G. Leishman, and S. L. Anderson. Stereo PIV measurements in the wake of a hovering rotor. In American Helicopter Society 56th Annal Forum, Virginia Beach, USA, May 2-4, 2000.

15. G. E. A. Meier. Computerized background-oriented Schlieren. Exp. Fluids, $3: 181,2002$.

16. M. Raffel, U. Seelhorst, and C. Willert. Recording and evaluation methods of PIV investigations on a helicopter rotor model. Exp. Fluids, 36:146, 2004.

17. M. Raffel, C. Tung, H. Richard, Y. Yu, and G. E. A. Meier. Background oriented stereoscopic schlieren (BOSS) for full scale helicopter vortex characterization. In 9th Int. Symp. on Flow Visualization, Edinburgh, UK, Aug. 22-25, 2000.

18. M. Raffel, C. Willert, S. Wereley, and J. Kompenhans. Particle image velocimetry, a practical guide. Springer, 2004.

19. H. Richard, J. Bosbach, A. Henning, M. Raffel, and B. G. van der Wall. 2C and 3C PIV measurements on a rotor in hover condition. In 13th International Symposium on Applications of Laser Techniques to Fluid Mechanics, Lisbon, Portugal, June 26-29, 2006.

20. H. Richard and M. Raffel. Visualization of vortical structures by density gradient detection. In PSFVIP-3, Maui, Hawaii, USA, June 18-21, 2001.

21. H. Richard and M. Raffel. Rotor wake measurements: full-scale and model tests. In American Helicopter Society 58th Annual Forum, Montréal, Canada, June 11-13, 2002.

22. B. G. van der Wall, C. L. Burley, Y. H. Yu, K. Pengel, and P. Beaumier. The HART II test - measurement of helicopter rotor wakes. Aerospace Sci. Tech., 8(4), 2004.

23. B. G. van der Wall and H. Richard. Analysis methodology for 3C-PIV data of rotary wing vortices. Exp. Fluids, 40:798, 2006.

24. G. H. Vatistas, V. Kozel, and W. C. Mih. A simpler model for concentrated vortices. Exp. Fluids, 11:73, 1991.

25. K. Y. Yick, R. Stocker, and T. Peacock. Microscale synthetic Schlieren. Exp. Fluids, 42(1):41, 2007. 